\title{
An Analytic Hedging Model of Energy Quanto Contracts
}

\author{
Sang Baum Kang, Jialin Zhao* \\ Stuart School of Business, Illinois Institute of Technology, Chicago, USA \\ Email: skang21@stuart.iit.edu, *jzhao28@hawk.iit.edu
}

How to cite this paper: Kang, S.B. and Zhao, J. (2017) An Analytic Hedging Model of Energy Quanto Contracts. Theoretical Economics Letters, 7, 737-746.

https://doi.org/10.4236/tel.2017.74053

Received: May 12, 2017

Accepted: June 4, 2017

Published: June 7, 2017

Copyright $\odot 2017$ by authors and Scientific Research Publishing Inc. This work is licensed under the Creative Commons Attribution International License (CC BY 4.0).

http://creativecommons.org/licenses/by/4.0/

\begin{abstract}
Earnings of energy firms are exposed to a joint risk of energy price and energy consumption. The correlated fluctuations in both price and consumption present a joint risk of price and volume for a load-serving entity (LSE). In order to manage such a joint risk, LSEs take positions in a variety of hedging contracts. Among these financial instruments, we analyze the use of electricity-temperature quantity-adjusting (quanto) contracts. In this paper, we consider an LSE that has access to electricity price derivatives, temperature derivatives, and energy quanto contracts. We derive the closed-form optimal hedging positions in these contracts and the optimal mean-variance tradeoff, from an analytic model that we develop within the Constant Absolute Risk Aversion (CARA)-normal setting. We mathematically prove that the use of quanto contracts allows an LSE to lower its revenue volatility. Furthermore, our model offers novel economic insights into the application of energy quanto contracts to hedging practice. First, we document and quantify the "dirty hedge" of standardized price and temperature derivatives in the absence of tailor-made energy quanto contracts. Second, we derive a threshold price of energy quanto contracts. If an energy quanto contract is quoted above this threshold price, an LSE shall not trade such a contract for risk management purposes. Third, this paper investigates a questionable, yet commonly adopted practice of using temperature as a perfect proxy for power consumption.
\end{abstract}

\section{Keywords}

Energy Quanto Contract, Financial Risk Management, Complex Derivative, Electricity Market, Energy Finance

\section{Introduction}

Earnings of energy firms are exposed to a joint risk of energy price and energy consumption. Take an electricity load-serving entity (LSE) as an example. A 
typical LSE charges its end-users a fixed retail price for all of their power consumption. When the demand exceeds its generation capacity, this LSE may need to purchase additional electricity from a wholesale market. The correlated fluctuations in both price and consumption present a joint risk of price and volume for this LSE.

In order to manage such a joint risk, LSEs take positions in a variety of hedging contracts. Among these financial instruments, this paper is particularly interested in the use of electricity-temperature quantity-adjusting (quanto) contracts. Quanto contracts originally appeared in currency-related markets, converting a certain amount of future financial transactions from one currency to another at a pre-stated exchange rate. Within the energy markets, quanto contracts generate cash settlements that depend on both energy prices and temperature. This mechanism of energy quanto contracts provides a channel to account for the indirect impact of energy consumption on energy prices. From this perspective, hedging using energy quanto contracts is more efficient than that using standardized price and temperature derivatives ${ }^{1}$ (see e.g., [1]). It is also worth noting that, unlike the aforementioned standardized instruments, energy quanto contracts are tailor-made and are illiquid in the markets.

In this paper, we consider an LSE that has access to electricity price derivatives, temperature derivatives, and energy quanto contracts. We derive the closedform optimal hedging positions in these contracts and the optimal mean-variance tradeoff, from an analytic model that we develop within the Constant Absolute Risk Aversion (CARA)-normal setting. We use the CARA-normal setting because it is analytically tractable, is consistent with a mean-variance framework, and is widely used in theoretical finance and economics (see [2] among many other papers).

We mathematically prove that the use of quanto contracts allows an LSE to lower its revenue volatility. Furthermore, our model offers novel economic insights into the application of energy quanto contracts to hedging practice. First, we document and quantify the "dirty hedge" of standardized price and temperature derivatives in the absence of tailor-made energy quanto contracts. Second, we derive a threshold price of energy quanto contracts. If an energy quanto contract is quoted above this threshold price, an LSE shall not trade such a contract for risk management purposes. Third, this paper investigates a questionable, yet commonly adopted practice of using temperature as a perfect proxy for power consumption.

Given the indirect impact of energy consumption on energy prices, it is not surprising that hedging using quanto contracts is more efficient than that using standardized price and temperature derivatives. [1] motivated the need for cross-market derivatives such as quanto contracts for efficient financial risk management. Recently [3] proposed an energy quanto pricing model based on Monte-Carlo simulation. In addition, [4] proposed an analytic model for quanto derivatives pricing. However, these models are mostly built upon risk-neutral

${ }^{1} \mathrm{~A}$ detailed discussion on price and temperature derivatives is presented in Section 2.1 . 
pricing and therefore are silent about a hedger's risk-aversion in an incomplete market. To fill this research gap, we propose a novel hedging model that does not depend on dynamic market completeness. Then, we uncover the aforementioned novel economic insights.

This paper contributes to the current literature of energy quanto contracts by proposing a hedging model that does not rest on dynamic market completeness. Existing models (e.g., [3] and [4]) are mostly built upon such assumptions. However, in an incomplete real-world market, the sellers of energy quanto contracts ask for high risk premia for the following reasons. First, tailor-made energy quanto contracts are illiquid in the markets. Second, energy quanto contracts are written on non-storable commodities (i.e., electricity and temperature), and thus, it is impossible or prohibitively expensive to replicate these instruments.

The remainder of this article is organized as follows. Section 2 presents our analytic hedging model of energy quanto contracts. Section 3 reports numerical examples, and Section 4 concludes.

\section{An Analytic Hedging Model of Energy Quanto Contracts}

\subsection{Hedging Instruments}

Power cannot be economically stored on a large scale. When power demand exceeds power generation, an LSE has to purchase electricity at a floating wholesale price and sells power at a fixed retail price. Earnings of this LSE can be written as $L \times(R-W)$, where $L$ stands for time-changing electricity consumption, $R$ stands for a fixed retail price, and $W$ stands for a floating wholesale price. The risk exposure of this LSE features a non-linear function on energy prices $(W)$ and load $(L)$.

Using price derivatives, this LSE can shelter its revenue from extreme changes in electricity prices. Payoffs of price derivatives are driven by floating wholesale prices $(W)$ in the power markets. As a result, these contracts lock in price risks for this LSE.

Temperature derivatives are used to hedge against volumetric risk because of the fact that temperature and power consumption are highly correlated. The most-traded temperature derivatives are written on Degree Day indices such as Heating Degree Day (HDD) and Cooling Degree Day (CDD).

Both electricity price derivatives and temperature derivatives are standardized and traded in the exchange markets. The common contract size of electricity price derivatives is 25 Mega-Watts, and the cash settlements of temperature derivatives have a notional value of $\$ 20$ per HDD or CDD tick. Unlike these standardized instruments, energy quanto contracts are tailor-made hedging deals. The payoffs of such contracts are driven by the product of energy prices and temperature indices. It is worth noting that energy quanto contracts are designed for specific firms and may vary in contract terms. One example of the payoffs of a typical energy quanto contract can be written as

$$
\text { Payoffs }_{\text {quanto }}=V \times\left(D-D_{f i x}\right) \times\left(W-W_{f i x}\right)
$$


where $V$ stands for the contract volume, $D(W)$ is the floating Degree Day index (electricity prices), and $D_{f i x}\left(W_{f i x}\right)$ is the pre-specified strike value of the Degree Day index (electricity prices).

Recall that the revenue of this LSE is a non-linear function on risk factors (e.g., energy prices and energy demand) and that the payoffs of energy quanto contracts account for the correlation between these risk factors. Energy quanto contracts take into consideration the indirect impacts of power demand on power prices. As a result, it is not a surprise that hedging using energy quanto contracts is more effective than that using standardized price and temperature derivatives (e.g., [1]).

\subsection{Economic Setting}

This paper considers a market setting in which an LSE has access to electricity price derivatives, temperature derivatives and energy quanto contracts. Accordingly, this LSE faces three underlying risk factors: temperature $(D)$, electricity prices $(W)$, and electricity load $(L)$. Specifically, $W$ and $L$ define the revenue, and $D$ and $W$ drive cash settlements from hedging contracts.

For analytic tractability, we assume that the aforementioned risk factors follow correlated normal distributions:

$$
\begin{gathered}
L=L_{0}+\sigma_{L}\left(\rho_{D L} \times Z_{1}+\sqrt{1-\rho_{D L}^{2}} \times Z_{2}\right) ; \\
W=W_{0}+\sigma_{W}\left(\rho_{D W} \times Z_{1}+\sqrt{1-\rho_{D W}^{2}} \times Z_{3}\right) ; \\
D=D_{0}+\sigma_{D} Z_{1} ;
\end{gathered}
$$

where $Z_{i}$ is a standard normal random variable, for $i=1,2$, and $3 ; L_{0}, W_{0}$ and $D_{0}$ stand for the expected mean of $L, W$ and $D ; \sigma_{L}, \sigma_{W}$, and $\sigma_{D}$ stand for the standard deviation of $L, W$ and $D ; \rho_{D W}$ stands for the correlation between $D$ and $W$; and $\rho_{D L}$ stands for the correlation between $D$ and $L$.

Using the modeling of underlying risk factors, we write the payoffs of this LSE. Recall that this LSE trades price derivatives, temperature derivatives, and energy quanto contracts. Because these instruments are written on non-storable underlying assets (e.g., electricity and temperature), convenience yields are not well defined. Thus, we follow [5] and build our modeling model on the expectations theory instead of the classic cost-of-carry theory. The expectations theory states that the forward price of a commodity equals the expected spot price of the underlying commodity during the delivery period, plus an expected risk premia.

Accordingly, an electricity forward price $F_{W}$ can be written as $F_{W}=E[W]+b_{W}$, a temperature forward price $F_{D}=E[D]+b_{D}$, and an energy quanto forward price $F_{Q}=E[W \times D]+b_{Q}$, with $b_{W}, b_{D}$ and $b_{Q}$ representing the risk premium (also referred to as the "bias" of electricity price, temperature, and quanto contracts). Such a bias reflects the hedging costs associated with trading corresponding financial instruments.

In addition, depending on the LSE's position in hedging devices, we construct four potential hedging portfolios. In order of sophistication, Strategy 1 is an un- 
hedged (naked) trading strategy; Strategy 2 involves only electricity derivatives; Strategy 3 employs both electricity and temperature contracts; and Strategy 4 further includes tailor-made energy quanto contracts. Let $V_{W}, V_{D}$ and $V_{Q}$ denote an LSE's hedging positions in electricity price derivatives, temperature derivatives, and energy quanto contracts, respectively. The payoffs $(\Pi)$ of each hedging portfolio can be summarized in Table 1 .

Using the mean-variance framework, we then develop a CARA-normal model to obtain new economic intuitions on the application of energy quanto contracts. In our model, an LSE's optimal hedging position $V_{W}^{*}, V_{D}^{*}$, and $V_{Q}^{*}$ shall satisfy:

$$
\max E[\Pi]-\frac{\gamma}{2} \operatorname{Var}[\Pi]
$$

where $\gamma$ is the risk-aversion parameter. Based on results by [6], we set $\gamma=3$ in this paper. Using the previously specified modeling of $L, W$ and $D$, we can derive $\Pi$ for each hedging portfolio in consideration, and we obtain a closed-form solution, accordingly.

\subsection{The Main Results}

For illustration purposes, we first solve a simplified case that sets $b_{W}, b_{D}$ and $b_{Q}$ $=0$; this case does not take into account the impact of contract bias. Table 2 reports the corresponding optimal solutions. Note that, in Table 2, we denote the linear regression coefficient of risk factor $a$ on $b$ as $\beta_{a / b}$ where $a, b=L, W$ and $D$. By analyzing the closed-form hedging solutions, we have the following observations:

Claim 1: The presence of energy quanto contracts allows an LSE to achieve lower revenue volatility.

Proof: Let $\operatorname{Var}_{3}^{*}$ be the variance of optimal payoffs of Strategy 3, and $\operatorname{Var}_{4}^{*}$ be that of Strategy 4. Our model implies that $\operatorname{Var}_{3}^{*}-\operatorname{Var}_{4}^{*}=\sigma_{L}^{2} \sigma_{W}^{2} \rho_{D L}^{2}\left(1-\rho_{D W}^{2}\right) \geq 0$.

Claim 2: When quanto contracts are not available, an LSE's optimal hedging positions in standardized derivatives contain a level of "dirty hedge", which function to mimic a quanto hedging. Such "dirty" hedges, however, do not provide a perfect risk control as quanto hedges do. Additionally, we quantify the amounts of "dirty hedge" as $\beta_{L / D} D_{0}$ for price contracts and $\beta_{L / D} W_{0}$ for temperature contracts.

Table 1. Payoffs of potential hedging portfolios.

\begin{tabular}{cc}
\hline Hedging Strategy & Payoffs Functions \\
\hline Strategy 1 & $L \times(R-W)$ \\
Strategy 2 & $L \times(R-W)+V_{W}\left(W_{i}-F_{W}\right)$ \\
Strategy 3 & $L \times(R-W)+V_{W}\left(W_{i}-F_{W}\right)+V_{D}\left(D_{i}-F_{D}\right)$ \\
Strategy 4 & $L \times(R-W)+V_{W}\left(W_{i}-F_{W}\right)+V_{D}\left(D_{i}-F_{D}\right)+V_{Q}\left(W_{i} \times D_{i}-F_{Q}\right)$ \\
\hline
\end{tabular}


Table 2. Analytic solution of the static hedging model (constrained case).

\begin{tabular}{cc}
\hline Panel A: Strategy 1 & Hedging Positions \\
\hline Electricity Contracts & 0 \\
Temperature Contracts & 0 \\
Energy Quanto Contracts & 0 \\
\hline Panel B: Strategy 2 & Hedging Positions \\
\hline Electricity Contracts & 0 \\
Temperature Contracts & 0 \\
Energy Quanto Contracts & $\beta_{L \mid W}\left(R-W_{0}\right)$ \\
\hline Panel C: Strategy 3 & Hedging Positions \\
\hline Electricity Contracts & $L_{0}$ \\
Temperature Contracts & $-\beta_{L \mid D}\left(R-W_{0}\right)$ \\
Energy Quanto Contracts & 0 \\
\hline Panel D: Strategy 4 & $\beta_{L \mid D}$ \\
\hline Electricity Contracts & $-\beta_{L \mid D} R$ \\
Temperature Contracts & $L_{L \mid D} D_{0}$ \\
Energy Quanto Contracts & Hositions \\
\hline
\end{tabular}

Proof: Let us then denote the optimal $V_{W}$ and $V_{D}$ of Strategy $i$ as $V_{W, i}^{*}$ and $V_{D, i}^{*}$ where $i=1,2,3$, and 4 . The amount of "dirty" hedges in price hedges equals $V_{W, 3}^{*}-V_{W, 4}^{*}=L_{0}-\left(L_{0}-\beta_{L \mid D} D_{0}\right)=\beta_{L \mid D} D_{0}$. Similarly, the amount of "dirty" hedges in temperature hedges equals $V_{D, 3}^{*}-V_{D, 4}^{*}=\beta_{L \mid D} W_{0}$.

We further incorporate hedging costs into our model by allowing for non-zero $b_{W}, b_{D}$ and $b_{Q}$. This case factors into the impact of contract bias on deriving optimal hedging solutions. The current paper refers to this case study as an "unconstrained case." The corresponding hedging solutions are presented in Table 3. It is easy to show that previous claims drawn under the zero bias (i.e., constrained case) still hold under this more general case.

In addition, we obtain useful economic insights into some interesting hedging issues. When trading energy quanto contracts, an LSE's optimal position in price contracts will hedge its price risks associated with an expected non-temperature-related load (a.k.a., base load) $L_{0}-\beta_{L \mid D} D_{0}$. This LSE's short position in temperature contracts will hedge its volume risks associated with retail revenue. Its long position in energy quanto contracts will hedge against this LSE's remaining price-volume joint risk.

We can also investigate the issues of using a temperature index as a perfect proxy for energy demand. Such a practice is a common assumption in the energy sector. Our analytic solutions show that optimal hedging positions in both temperature and energy quanto contracts depend on $\beta_{L / D}$. Compared to using assumptions of perfectly correlated $D$ and $L$ (i.e., $\beta_{L / D}=1$ ), an LSE realizing that 
Table 3. Analytic solution of the static hedging model (unconstrained case).

\begin{tabular}{|c|c|}
\hline Panel A: Strategy 1 & Hedging Positions \\
\hline Electricity Contracts & 0 \\
\hline Temperature Contracts & 0 \\
\hline Energy Quanto Contracts & 0 \\
\hline Panel B: Strategy 2 & Hedging Positions \\
\hline Electricity Contracts & $V_{W, \text { Strategy } 2}^{*}-b_{W} / \gamma / \sigma_{W}^{2}$ \\
\hline Temperature Contracts & 0 \\
\hline Energy Quanto Contracts & 0 \\
\hline Panel C: Strategy 3 & Hedging Positions \\
\hline Electricity Contracts & $V_{W, \text { Srategy } 3}^{*}+\frac{1}{\gamma \sigma_{D} \sigma_{W}^{2}\left(\rho_{D W}^{2}-1\right)}\left(b_{W} \sigma_{D}-b_{D} \sigma_{w} \rho_{D W}\right)$ \\
\hline Temperature Contracts & $V_{D, \text { Strategy } 3}^{*}+\frac{1}{\gamma \sigma_{W} \sigma_{D}^{2}\left(\rho_{D W}^{2}-1\right)}\left(b_{D} \sigma_{W}-b_{W} \sigma_{D} \rho_{D W}\right)$ \\
\hline Energy Quanto Contracts & 0 \\
\hline Panel D: Strategy 4 & Hedging Positions \\
\hline Electricity Contracts & $\begin{array}{l}V_{W, \text { Strategy } 4}^{*}-\frac{1}{\gamma \sigma_{D}^{2} \sigma_{W}^{2}\left(\rho_{D W}^{4}-1\right)}\left(D_{0} b_{Q}-\sigma_{D}^{2} b_{W}-D_{0}^{2} b_{W}\right. \\
-D_{0} b_{Q} \rho_{D W}^{2}-D_{0} W_{0} b_{D}-\sigma_{0}^{2} b_{W} \rho_{D W}^{2}-D_{0}^{2} b_{W} \rho_{D W}^{2} \\
\left.+\sigma_{D} \sigma_{W} b_{D} \rho_{D W}+\sigma_{D} \sigma_{W} b_{D} \rho_{D W}^{3}+D_{0} W_{0} b_{D} \rho_{D W}^{2}\right)\end{array}$ \\
\hline Temperature Contracts & $\begin{array}{l}V_{D, \text { Strategy } 4}^{*}-\frac{1}{\gamma \sigma_{D}^{2} \sigma_{W}^{2}\left(\rho_{D W}^{4}-1\right)}\left(W_{0} b_{Q}-\sigma_{W}^{2} b_{D}-W_{0}^{2} b_{D}\right. \\
-W_{0} b_{Q} \rho_{D W}^{2}-D_{0} W_{0} b_{W}-\sigma_{W}^{2} b_{D} \rho_{D W}^{2}-W_{0}^{2} b_{D} \rho_{D W}^{2} \\
\left.+\sigma_{D} \sigma_{W} b_{W} \rho_{D W}+\sigma_{D} \sigma_{W} b_{W} \rho_{D W}^{3}+D_{0} W_{0} b_{W} \rho_{D W}^{2}\right)\end{array}$ \\
\hline Energy Quanto Contracts & $V_{Q, \text { Srrategy } 4}^{*}-\frac{1}{\gamma \sigma_{D}^{2} \sigma_{W}^{2}\left(\rho_{D W}^{4}+1\right)}\left(D_{0} b_{w}-b_{Q}+W_{0} b_{D}\right)$ \\
\hline
\end{tabular}

$D$ and $L$ are not perfectly correlated (i.e., $\beta_{L / D}<1$ ) will reduce its positions in temperature contracts and energy quanto contracts. On the other hand, this LSE's optimal position in electricity price derivatives is immune to this imperfect proxy issue.

Furthermore, our model sheds light on the fair pricing of energy quanto contracts. The underlying principle of our pricing is very intuitive. Assuming that this LSE trades financial contracts for hedging purposes only, it will not enter positions in energy quanto contracts if hedging costs are extremely high.

Claim 3: In the risk-hedging context, if energy quanto contracts are too expensive

$$
b_{Q}>D_{0} b_{W}+W_{0} b_{D}+\gamma \sigma_{D} \rho_{D L} \sigma_{W}^{2}\left(\rho_{D W}^{2}+1\right)
$$

a rational LSE does not trade any quanto contract.

Proof: Let us denote the optimal $V_{Q}$ of Strategy $i$ under the unconstrained case as $V_{Q, i}^{U C}$ and under the constrained (simplified) case as $V_{Q, i}^{C}$, where $i=1,2$, 
3 , and 4 . The deviation of the amount of quanto hedges between the unconstrained and constrained cases is the following.

$$
V_{Q, 4}^{* C}-V_{Q, 4}^{* U C}=\frac{1}{\gamma \sigma_{D}^{2} \sigma_{W}^{2}\left(\rho_{D W}^{2}+1\right)}\left(D_{0} b_{W}-b_{Q}+W_{0} b_{D}\right)
$$

When $b_{Q}>D_{0} b_{W}+W_{0} b_{D}$, an LSE needs to reduce its position in energy quanto contracts by the amount specified in Equation 1.7. Recall that this LSE can only lower its energy quanto positions by as much as $V_{Q, 4}^{* U C}$. A further reduction results in a short position in energy quanto contracts, which is against this LSE's goal of risk hedging. Therefore, for an LSE to buy energy quanto contracts for hedging purposes, the market should satisfy the following requirement.

$$
\left|\frac{1}{\gamma \sigma_{D}^{2} \sigma_{W}^{2}\left(\rho_{D W}^{2}+1\right)}\left(D_{0} b_{W}-b_{Q}+W_{0} b_{D}\right)\right| \leq V_{Q, 4}^{* C}
$$

That is,

$$
b_{Q} \leq D_{0} b_{W}+W_{0} b_{D}+\gamma \sigma_{L} \sigma_{D} \rho_{L D} \sigma_{W}^{2}\left(\rho_{D W}^{2}+1\right)
$$

\section{Numerical Examples}

For illustration purposes, our numerical examples consider fictitious electricity LSE serving the Chicago area. We assume that this LSE is under contract to supply one percent of the PJM system load with a fixed retail rate Rat $\$ 45.97 /$ MWh quoted from the Commonwealth Edison Rate Book.

Recall from our previous discussion on hedging payoffs (see Table 1) that this LSE faces three underlying risk factors: temperature, electricity load and electricity prices. Accordingly, we collect data, including Chicago temperatures from the U.S. National Climatic Data Center, electricity loads from the PJM electricity market, and electricity day-ahead prices from the PJM North Illinois Hub. We model these risk factors using Equations 1.2, 1.3, and 1.4 and then derive optimal hedging solutions by solving the mean-variance framework specified in Equation 1.5. Table 4 presents the corresponding results.

This numerical example reconfirms our insights drawn from the analytic hedging model of energy quanto contracts. In order to hedge against its pricevolume joint risk, an LSE takes a long position in electricity price contracts, a short position in temperature contracts, and a long position in energy quanto

Table 4. Numerical optimal solutions (constrained case).

\begin{tabular}{ccccc}
\hline $\begin{array}{c}\text { Hedging } \\
\text { Strategy }\end{array}$ & $\begin{array}{c}\text { Electricity Price } \\
\text { Contracts } \\
(\mathrm{MWh})\end{array}$ & $\begin{array}{c}\text { Temperature } \\
\text { Contracts } \\
(\$ / \mathrm{DD})\end{array}$ & $\begin{array}{c}\text { Energy Quanto } \\
\text { Contracts } \\
(\mathrm{MWh} / \mathrm{DD})\end{array}$ & $\begin{array}{c}\text { Mean-Variance } \\
\text { Measure } \\
\text { (million, \$) }\end{array}$ \\
\hline Strategy 1 & 0 & 0 & 0 & $-5,364,339$ \\
Strategy 2 & 106,846 & 0 & 0 & $-136,864$ \\
Strategy 3 & 107,310 & -1650 & 0 & $-136,784$ \\
Strategy 4 & 91,055 & $-85,542$ & 1860 & $-27,191$ \\
\hline
\end{tabular}


contracts. When energy quanto contracts are not accessible (i.e., Strategy 3), this LSE increases its long position in price derivatives and reduces its short position in temperature derivatives by $16,255 \mathrm{MWh}$ and $\$ 83,892 / \mathrm{DD}$, respectively. These adjustments in hedging positions reflect the need to use standardized price and temperature derivatives to mimic the function of energy quanto contracts. This observation agrees with Claim 1, in that the introduction of energy quanto contracts provides more efficient hedges than standardized price and temperature derivatives.

Such a "dirty hedge" of standardized contracts is still less efficient than the use of energy quanto contracts. When hedging costs are not taken into consideration, the hedging performance of Strategy 3 (i.e., using standardized instruments), which is measured by the resulting mean-variance, is lower than that of Strategy 4 (using both standardized instruments and energy quanto contracts).

\section{Conclusions}

In this paper, we develop an analytic hedging model of energy quanto contracts and investigate several interesting hedging issues related to the application of such financial instruments. Of particular interest to practitioners, we document that, when energy quanto contracts are not available, an LSE shall use standardized price and temperature derivatives to mimic the function of energy quanto contracts. Our paper further quantifies such a "dirty hedge" of standardized financial instruments in both the analytic hedging model and the numerical examples.

In addition, we investigate a questionable, yet commonly adopted practice in risk management. That is, an LSE may use temperature as a perfect proxy for power consumption. Given that temperature and power demand are not perfectly correlated, our model offers the following guidance to an LSE: an LSE should reduce its positions in temperature derivatives and energy quanto contracts, but should maintain the same position in price derivatives.

The current research opens a new avenue for future research. First, to study energy quanto derivatives, an energy finance researcher may consider stylized facts of electricity price, load, and temperature time series, such as seasonality, time-varying variance, and the time-lagged dependence (see, e.g., [7]). Then, she/he may lose the analytic tractability of our CARA-normal framework, but can still use Monte-Carlo simulation to calculate hedge effectiveness of quanto derivatives in a more realistic market setting. The results from the model in this paper will give guidance in interpreting such Monte-Carlo simulation results. Second, our model may be applicable to other financial markets such as foreign currency and agricultural markets. We leave these interesting topics as a future research agenda.

\section{Acknowledgements}

We thank participants at 2014 International Association of Energy Economics Asian Conference (Beijing, China) and at the Friday Research Seminar of Illinois 
Institute of Technology for their relevant questions and useful comments. We thank Dr. Caroline Toscano for proofreading. All errors are strictly ours.

\section{References}

[1] Ho, T.S., Stapleton, R.C. and Subrahmanyam, M.G. (1995) Correlation Risk, Cross-Market Derivative Products and Portfolio Performance. European Financial Management, 1, 105-124. https://doi.org/10.1111/j.1468-036X.1995.tb00011.x

[2] Ozsoylev, H. and Werner, J. (2011) Liquidity and Asset Prices in Rational Expectations Equilibrium with Ambiguous Information. Economic Theory, 48, 469-491. https://doi.org/10.1007/s00199-011-0648-0

[3] Caporin, M., Preś, J. and Torro, H. (2012) Model Based Monte Carlo Pricing of Energy and Temperature Quanto Options. Energy Economics, 34, 1700-1712.

[4] Benth, F.E., Lange, N. and Myklebust, T.A. (2015) Pricing and Hedging Quanto Options in Energy Markets. Journal of Energy Markets, 8, 1-35. https://doi.org/10.21314/JEM.2015.130

[5] Bessembinder, H. and Lemmon, M.L. (2002) Equilibrium Pricing and Optimal Hedging in Electricity forward Markets. Journal of Finance, 57, 1347-1382. https://doi.org/10.1111/1540-6261.00463

[6] Bliss, R.R. and Panigirtzoglou, N. (2004) Option-Implied Risk Aversion Estimates. Journal of Finance, 59, 407-446. https://doi.org/10.1111/j.1540-6261.2004.00637.x

[7] Zhao, J. and Kang, S.B. (2015) Co-Simulation of Risk Factors in Power Markets. Energy Risk, 44-49. https://doi.org/10.2139/ssrn.2614664

\section{Scientific Research Publishing}

\section{Submit or recommend next manuscript to SCIRP and we will provide best} service for you:

Accepting pre-submission inquiries through Email, Facebook, LinkedIn, Twitter, etc. A wide selection of journals (inclusive of 9 subjects, more than 200 journals) Providing 24-hour high-quality service User-friendly online submission system Fair and swift peer-review system Efficient typesetting and proofreading procedure Display of the result of downloads and visits, as well as the number of cited articles Maximum dissemination of your research work

Submit your manuscript at: http://papersubmission.scirp.org/ Or contact tel@scirp.org 